\title{
CARACTERÍSTICAS FÍSICAS DEL RESERVORIO EN EL CAMPO GEOTÉRMICO LAS PAILAS \\ ESTIMADAS CON BASE EN DATOS SISMOLÓGICOS, ESTRUCTURALES Y RELACIONES DE POISSON $(\sigma)$
}

\author{
Waldo Taylor \\ Área de Amenazas y Auscultación Sísmica y Volcánica \\ Instituto Costarricense de Electricidad (ICE) \\ E-mail: wtaylor@ice.go.cr
}

(Recibido 9/12/03; Aceptado 3/2/04)

\begin{abstract}
During the second half of 2002, a seismic swarm (more than 250 microearthquakes) was recorded at Las Pailas Geothermal Field (PGF) with local magnitude $\mathrm{M}_{\mathrm{L}}<2.0$ and depths between 1.0 and $5.5 \mathrm{~km} .116$ earthquakes were located and 86 were used to draw Poisson ratio $(\sigma)$ maps. Using seismic distribution, structural data, polarization of the fastest $\mathrm{S}$ wave and field observations, I determined, identified and verified faults and fractures with NW and NE directions, where two of them are strike slip active faults. Based on Poisson ratios, data field and seismicity analysis, I conclude that PGF is a high-pressure water domain reservoir controlled by fault systems and can be divided in sectors according to three different conditions or classifications. The first condition has low $\sigma$ values (higher vapor amount) and low permeability, is located to the east and west limits of PGF, where the PGP-02 and PGP-05 wells are located. The second condition has high $\sigma$ values (between 0.24 and 0.30 ) normally suggesting higher fluids amount, within this sector wells PGP-04 and PGP06 are located. The third sector has intermediate or higher $\sigma$ values $(0.18-0.23)$ and can be related with phase shift areas along fault systems and where wells PGP-01 and PGP-03 are located.
\end{abstract}

Key words: geothermal reservoir, Poisson, seismicity, Las Pailas, Costa Rica.

RESUMEN: Durante el segundo semestre del 2002, en el Campo Geotérmico Las Pailas (CGP), se registró un enjambre sísmico (más de 250 microsismos) con magnitudes locales $\mathrm{M}_{\mathrm{L}}<2,0$ y profundidades entre 1,0 y $5,5 \mathrm{~km}$. Fue posible localizar 116 sismos y de esos se utilizaron 86 para confeccionar los mapas de isovalores de relación de Poisson $(\sigma)$. Utilizando datos estructurales, la polarización de la onda $\mathrm{S}$ más rápida y observaciones de campo, se determinó, identificó y se verificó la existencia de fallas y fracturas de rumbos $\mathrm{NO}$ y NE, que por lo general son de tipo desplazamiento de rumbo, y que de acuerdo con la sismicidad estudiada, al menos dos de ellas son activas. Con base en las relaciones de Poisson, datos de campo y análisis de la sismicidad, se estima que el CGP es un reservorio de alta presión dominado por agua, con un control estructural, que puede ser dividido en sectores, según tres diferentes condiciones o clasificaciones. La primera 
condición presenta valores bajos de $\sigma$ (mayor contenido de vapor) y poca permeabilidad, se localiza al este y al oeste del CGP y en ellas se encuentran los pozos PGP-05 y PGP-02. La segunda condición tiene valores altos de $\sigma$ (entre 0,24 y 0,30 ), es decir, el contenido de líquidos es mayor, justo donde se encuentran los pozos PGP-04 y PGP-06. La tercera condición posee valores de $\sigma$ intermedios $(0,18-0,23)$ donde se puede dar el cambio de fases y por lo general se distribuyen a lo largo de las zonas de fallas, en donde se localizan los pozos PGP-01 y PGP-03.

Palabras clave: reservorio geotérmico, Poisson, sismicidad, Las Pailas, Costa Rica.

\section{INTRODUCCIÓN}

En este trabajo se integraron el análisis de los datos sismológicos, estructurales y las observaciones de trabajo de campo (como el grado de fracturación de las rocas y la orientación de las fracturas y los paleocauces), con el fin inmediato de caracterizar el Campo Geotérmico Las Pailas (CGP), desde el punto de vista sismotectónico, utilizando los valores de las relaciones de Poisson, como una medida indirecta de las propiedades físicas de las rocas.

Los microsismos son usados para determinar la orientación de las fallas activas y el tipo de fallamiento por medio de mecanismos focales. El éxito depende del uso de un modelo de velocidades adecuado y correcto y la buena calidad de los datos obtenidos durante el enjambre del año 2002 en el sector de las Pailas, que permitieron la definición de un modelo inicial.

La corteza terrestre es un medio anisotrópico que afecta la velocidad de las ondas cuando estas pasan a través de capas, alineamientos de granos o cristales, fallas, microfracturas y espacios de poros orientados. De todas ellas, en la mayoría de los casos, la orientación de fallas y microfracturas, son las que tienen el mayor efecto $y$ hace que las velocidades de la cortante (onda S) se desplacen a diferentes velocidades. Por esa razón, es importante delimitar estructuras geológicas que pueden afectar las propiedades físicas dentro del reservorio. Tales estructuras son barreras importantes o lugares por donde se movilizan o se acumulan los fluidos.

Cuando las ondas cortantes (ondas S) tienen movimientos de partícula paralelos al rumbo de las fallas o fracturas, viajan a mayor velocidad que aquellas que se desplazan en la dirección ortogonal (Peacok et al., 1988; Kaneshima, 1990; Aster \& Shearer, 1992). Aunque existen varios métodos y técnicas para determinar la dirección de polarización de la onda cortante más rápida, en este estudio se aplicó el método visual del movimiento de partícula para determinar dicho ángulo.

Una vez definido el modelo de corteza a utilizar, localizar los sismos y determinar la orientación de las fracturas, era importante observar algunas de estas fallas y fracturas en el campo para la respectiva confirmación de los resultados. Por último, es importante dividir el CGP según la distribución espacial de las regiones que se interpretan con mayor contenido de vapor, mayor contenido de líquidos y la zona de intercambio de fases.

Uno de los aspectos más críticos en los sistemas geotérmicos, es determinar dónde ocurre la separación de las fases líquido-gas. Esto depende de la temperatura, la presión, la densidad de fracturas saturadas y la composición química de los fluidos. A poca profundidad en la corteza, la separación de fases comúnmente produce dos tipos de reservorios, en donde uno es dominado por líquidos y en el otro predomina el vapor.

La separación de fases aumenta las corrientes de convección y eso moviliza el calor más eficientemente que la circulación de fluidos por sí sola, es decir, la separación de fases acelera el enfriamiento de las fuentes de calor. Los sistemas geotermales en los cuales la separación de fases no ha ocurrido, perduran 10 veces más que aquellos en donde es evidente la separación de fases (Ito et al., 1979). Por esa razón es importante y necesario saber si la separación de fases se ha iniciado en el reservorio de un campo geotérmico explotable o en explotación y cuánto puede afectar la vida útil del proyecto. El método usado para conocer dónde se localizan las zonas con 
mayor o menor contenido de fluidos o vapor, es obteniendo la relación entre las velocidades de ondas P y $\mathrm{S}\left(\mathrm{V}_{\mathrm{P}} / \mathrm{V}_{\mathrm{S}}\right)$ y la relación de Poisson $(\sigma)$, debido a que estas están directamente relacionadas con las propiedades físicas de la roca, tales como la compresibilidad.

La relación de Poisson se define como la relación que hay entre la deformación del eje transversal y la deformación del eje longitudinal en la dirección en la cual se aplica una fuerza. Normalmente, los materiales comunes tienen valores de $\sigma$ que pueden variar entre 0,0 y 0,5 y está fuertemente relacionado con los módulos elástico, volumétrico y de corte. La relación de Poisson de los materiales influencia la velocidad de propagación de las ondas sísmicas. En aplicaciones geológicas, la relación entre las velocidades de la onda compresional (onda P) y la onda de corte (onda S), es importante para inferir la naturaleza y el estado de la roca en la profundidad.

O’Connel \& Budiansky (1977) demostraron que el módulo de corte de las rocas no es afectado por la saturación de agua, mientras que el módulo volumétrico es altamente sensitivo. Esto implica que las variaciones de $\mathrm{V}_{\mathrm{P}} / \mathrm{V}_{\mathrm{S}}$ y $\sigma$ están más controladas por la velocidad de la onda $\mathrm{P}\left(\mathrm{V}_{\mathrm{P}}\right)$ que por la velocidad de la onda $\mathrm{S}\left(\mathrm{V}_{\mathrm{S}}\right)$ en un reservorio dominado por líquido. Ito et al. (1979) señalan que los espacios de poro rellenados por agua y vapor afectan la transmisión de las ondas $\mathrm{P}$ y $\mathrm{S}$ en forma diferente. La relación de $\mathrm{V}_{\mathrm{P}} / \mathrm{V}_{\mathrm{S}}$ y $\sigma$ se incrementan desde una condición saturada de vapor (baja presión de poro) a una condición saturada de líquido (alta presión de poro). Estas observaciones son soportadas por estudios que demuestran que los sistemas dominados por agua como en East Mesa en California y Cerro Prieto en México, tienen altos valores de $\sigma$ (entre 0,2 y 0,3). Los sistemas dominados por vapor como The Geysers y Coso Hot Springs en California, tienen valores bajos de $\sigma$, entre 0,15 y 0,20 (Majer \& McEvilly, 1979). Julian et al. (1996) también demostraron que altas concentraciones de $\mathrm{CO}_{2}$ en el reservorio conducen a valores bajos de $\sigma$.

Basados en lo anteriormente señalado, los principales objetivos de este estudio fueron mejorar el modelo de velocidades en la corteza, identificar las fallas activas y los sistemas de fracturas, y determinar la distribución espacial de las relaciones de Poisson $(\sigma)$ en el CGP y su posible relación con parámetros físicos de temperaturas y presión (mayor contenido de vapor o agua) usando datos sismológicos obtenidos durante setiembre del año 2002. Además, durante el segundo semestre del año 2003 se realizó trabajo de campo para verificar la existencia de estructuras geológicas que coincidieran con la distribución de los sismos y las curvas de isovalores de Poisson.

Barahona et al. (2001) sugieren que el área de estudio hay una estructura caldérica con un alto grado de erosión en el borde, rellenada por depósitos posteriores a su formación, por lo que su escarpe no alcanza alturas mayores a los 40 metros. Arias (2002) propuso que es una tectocaldera, con orientación NO-SE, controlada por fallas de desplazamiento de rumbo sinestrales, que forman una estructura en gradas que se profundiza de suroeste a noreste, y que pueden estar asociadas de manera directa con la Falla Longitudinal de Costa Rica, que es una paralela a la fosa. Existen también fallas de rumbo NE, relacionadas con eventos más recientes y que son fallas de segunda generación, sintéticas y antitéticas producto del movimiento sinestral de las fallas maestras de rumbo NO-SE, que hacen que la tectónica dentro y en los alrededores del CGP sea compleja.

Geológicamente en el área aflora lo que se ha denominado Unidad Guachipelín, que consiste en secuencias de depósitos que presentan al menos cuatro facies: de caída, de flujo piroclástico, de flujo de pómez y de oleadas, por lo que es común observar estratos con espesores que pueden variar de pocos centímetros a metros (Barahona et al., 2001).

\section{METODOLOGÍA}

\section{Modelo de velocidades de la corteza}

El primer paso fue calcular el modelo de velocidades de la corteza con el fin de obtener la mejor localización posible de los sismos del enjambre sísmico del 2002. Esto se realizó utilizando el programa de modelos múltiples incluido 
en el programa SEISAN (Havskov \& Ottemöller, 2000). Este programa consiste en localizar los sismos usando diferentes modelos de velocidades en la corteza, partiendo de un modelo inicial. Las velocidades y la profundidad de las capas se van variando y el mejor modelo de corteza es aquel cuyo resultado provea la mínima raíz media cuadrática (rms, por su sigla en inglés).

\section{Distribución espacial de los sismos}

Se obtuvieron los tiempos de arribo de las ondas primarias $(\mathrm{P})$ y las secundarias $(\mathrm{S})$ y se localizaron los sismos utilizando el programa SEISAN (Havskov \& Ottemöller, 2000), para obtener latitud, longitud y profundidad para definir la orientación y el tipo de fallamiento usando mecanismos focales compuestos.

\section{Orientación de las fracturas usando las ondas sísmicas cortantes}

Para el análisis de la orientación de las fracturas se utilizó el programa PITSA (Scherbaum et al., 1999). El procesamiento consistió en suavizar y remuestrear las señales a 500 muestras por segundo (mps) y filtrar la señal utilizando el filtro pasabanda de Butterworth entre 1 y $15 \mathrm{~Hz}$ (el registro original tenía $100 \mathrm{mps}$ y la onda $\mathrm{S}$ tiene frecuencias entre 1 y $15 \mathrm{~Hz}$ ). Ambos pasos son utilizados para una mejor identificación y medida de la dirección del ángulo de la polarización más rápida (f). Después se procedió al análisis del movimiento horizontal de la partícula usando las componentes $\mathrm{N}$ y E del sensor y se determinó $\mathrm{f}$.

\section{Trabajo de campo}

Una vez obtenidas las direcciones de las fracturas y fallas por medio de los datos sismológicos (según el procedimiento descrito arriba), se realizaron seis días de trabajo de campo y el análisis de fotografías aéreas, con el fin de verificar la existencia de estas fallas y fracturas en superficie o en las capas inferiores y confirmar que las direcciones obtenidas con los datos sismológicos fueran correctas.

\section{Cálculo de las relaciones de Poisson}

Para el cálculo de $\mathrm{V}_{\mathrm{P}} / \mathrm{V}_{\mathrm{S}}$ se utilizó el método de Wadati (1933), en donde los tiempos de llegada de las ondas $\mathrm{P}$ y $\mathrm{S}$ pueden ser leídos en dos o más estaciones y la relación $\mathrm{V}_{\mathrm{P}} / \mathrm{V}_{\mathrm{S}}$ y el tiempo origen pueden ser calculados. Si se grafica el tiempo de llegada de $\mathrm{S}$ contra el tiempo de llegada de $\mathrm{P}$, la recta de mejor ajuste entre los puntos define una pendiente que está asociada directamente con la relación $\mathrm{V}_{\mathrm{P}} / \mathrm{V}_{\mathrm{S}}$.

La ventaja de este método es que el cálculo de la pendiente es independiente del modelo de corteza utilizado. Una vez obtenidos estos valores, se procedió al cálculo de la relación de Poisson $(\sigma)$ utilizando la siguiente fórmula:

$$
\begin{gathered}
\sigma=\left(\mathrm{K}^{2}-2\right) / 2\left(\mathrm{~K}^{2}-1\right) \\
\text { donde } \mathrm{K}=\mathrm{V}_{\mathrm{P}} / \mathrm{V}_{\mathrm{S}}
\end{gathered}
$$

Los mapas de isovalores de $\sigma$ fueron construidos usando el programa Surfer 7.0 y los datos fueron tratados estadísticamente usando un procedimiento de estimación llamado "kriging" (Krige, 1966).

Como los datos analizados están en función de la distancia, la base de datos final tendrá valores asignados que estarán distribuidos de acuerdo con la posición geográfica, es decir, cada valor calculado está definido por latitud, longitud y profundidad.

\section{RESULTADOS}

\section{Cálculo del modelo de velocidades de la corteza}

Para este estudio se usó el programa de modelos múltiples (Havskov \& Ottemöller, 2000), y el modelo de velocidades inicial de 
entrada utilizado fue el de Miravalles (Taylor, 2000). Se localizaron los sismos utilizando más de 1000 modelos de velocidades diferentes. Sin embargo, debido a que las profundidades de los sismos utilizados no superaron los $5,5 \mathrm{~km}$, el cambio en el modelo de corteza solo se realizó en esos primeros kilómetros. El cuadro 1 muestra el modelo de velocidades inicial utilizado (modelo de velocidades en Miravalles) y el modelo de velocidades que dio el menor rms $(0,061)$.

\section{Análisis de la distribución espacial de los sismos}

Una vez definido el modelo de corteza a utilizar, se procedió a la localización de los sismos. El área del CGP estudiada (7 km x $8 \mathrm{~km})$ cuenta con una base de datos de más de 250 microsismos, registrados durante el enjambre sísmico de setiembre del año 2002, de los cuales fue posible localizar $116\left(\mathrm{M}_{\mathrm{L}}<2,0\right)$, con profundidades entre 1,0 y $5,5 \mathrm{~km}$ (83 sismos están entre 1 y $2 \mathrm{~km}$ de profundidad). La figura 1 muestra la distribución espacial de los sismos.

La actividad sísmica registrada se inició muy cerca de la Hacienda Guachipelín y del pozo PGP-05. La mayoría de los sismos se registraron durante los días 4 y 5 de setiembre en un período de 20 horas. Conforme transcurrieron los días, la sismicidad se fue desplazando hacia el noreste (la actividad sísmica se concentró en los días 20, 21 y 22 de setiembre), volviendo inestable toda el área. La complejidad tectónica del área según Arias (2002) y explicada brevemente en la introducción del presente estudio, sugiere que el enjambre sísmico responde a una actividad sísmica asociada con las fallas existentes. Como se aprecia en la figura 1, la mayoría de los microsismos se localizan dentro de la caldera de Guachipelín, y especialmente muy cerca de las intersecciones de varias fallas y bordes de caldera, especialmente a lo largo de dos sistemas de fallas, el primero que se extiende desde el norte de la Hacienda Guachipelín (HG) y tiene rumbo NO-SE y el segundo sistema se inicia desde la HG con rumbo NE-SO. Sin embargo, no se descarta la posibilidad de que la sismicidad fuera disparada por las actividades de perforación de los pozos a lo largo de las zonas de falla, especialmente cerca del pozo PGP-05.

En la figura 2 se muestra la localización de los sismos registrados en las cuatro estaciones sismológicas instaladas en el área de Borinquen-Las Pailas (25 sismos que representan las localizaciones más confiables y de mejor calidad). En la figura también se muestra el mecanismo focal compuesto usando estos sismos, y sugiere la existencia de un sistema de falla con rumbos que coinciden con los resultados obtenidos en los análisis de los datos estructurales (Barahona et al., 2001 y Arias, 2002), fotografías aéreas, polarización de ondas cortantes y observaciones de campo.

Cuadro 1

Modelo de velocidades final usado en la localización de los sismos en el CGM y en el CGP

\begin{tabular}{cccccc}
\hline \multicolumn{3}{c}{ Modelo de Miravalles } & \multicolumn{3}{c}{ Modelo de Las Pailas } \\
\hline Profundidad (km) & VP (km/s) & VS (km/s) & Profundidad (km) & VP (km/s) & VS (km/s) \\
\hline $0,0-3,2$ & 3,70 & 2,14 & $0,0-2,5$ & 3,70 & 2,14 \\
$3,2-7,2$ & 5,90 & 3,41 & $2,5-7,2$ & 5,50 & 3,18 \\
$7,2-16,0$ & 6,40 & 3,70 & $7,2-16,0$ & 6,40 & 3,70 \\
$16,0-41,0$ & 6,60 & 3,82 & $16,0-41,0$ & 6,60 & 3,82 \\
$41,0-80,0$ & 7,90 & 4,57 & $41,0-80,0$ & 7,90 & 4,57 \\
$>80,0$ & 8,20 & 4,74 & $>80,0$ & 8,20 & 4,74 \\
\hline
\end{tabular}




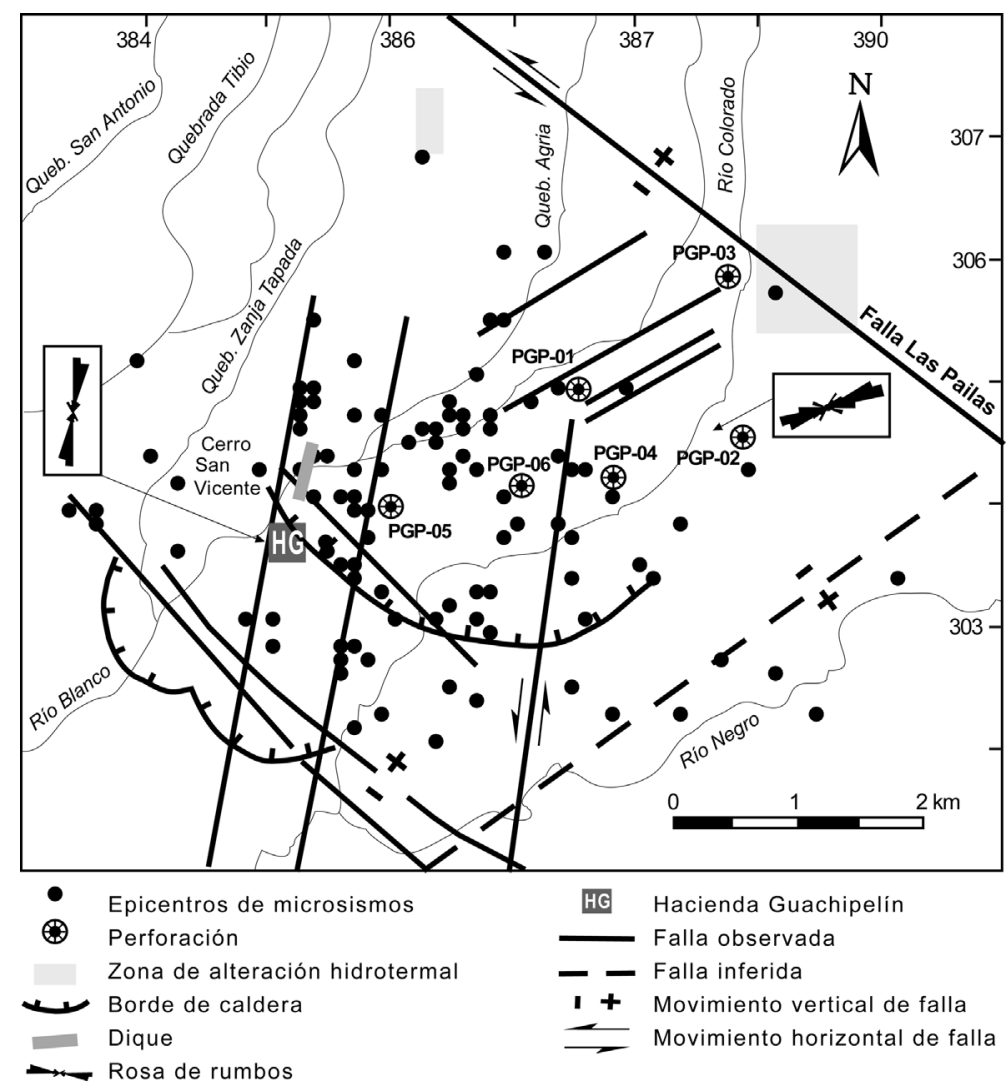

Fig. 1: Localización de los microsismos en el área del CGP (modificado de Arias, 2002).

Determinación de la orientación de las fracturas usando las ondas sísmicas cortantes

Se estudió la anisotropía sísmica al este y al oeste del CGP usando los datos digitales de las ondas cortantes (ondas S), registradas en dos estaciones (HGUA y HRDV) localizada la primera en la Hacienda Guachipelín y la segunda en las cercanías del pozo PGP-02 en la entrada de la Hacienda Rincón de la Vieja (Fig. 2; cuadro 2). Se analizaron los datos de las señales que tenían una buena amplitud y un claro arribo de las ondas S, para mejor identificación de la dirección de polarización (f) de la onda cortante más rápida. El cuadro 2 muestra un resumen de las estadísticas obtenidas con los datos analizados.

Estos resultados sugieren que el CGP tiene fracturas con rumbos que se orientan de ENE en el sector este del campo geotérmico, a rumbos NNE en el sector oeste. En la figura 1 se incluyen los diagramas de rosas obtenidos en los dos sitios y es clara la buena correlación con las fallas y fracturas observadas en el campo (ver análisis de los datos de campo) y con los patrones de drenaje (especialmente de los ríos Blanco y Colorado).

Cuadro 2

Dirección promedio de la polarización (f) de la onda S más rápida obtenida en las estaciones HGUA y HRDV

\begin{tabular}{|c|c|c|c|}
\hline Estación & Localización & $\begin{array}{c}\text { Cantidad } \\
\text { de datos } \\
\text { analizados }\end{array}$ & $\begin{array}{c}\text { Rumbo } \\
\text { (f) promedio } \\
\text { predominante }\end{array}$ \\
\hline HGUA & $303,7 \mathrm{~N} / 385,2 \mathrm{E}$ & 92 & $\mathrm{~N} 11^{\circ} \mathrm{E}$ \\
\hline HRDV & $304,6 \mathrm{~N} / 388,7 \mathrm{E}$ & 83 & $\mathrm{~N} 66^{\circ} \mathrm{E}$ \\
\hline
\end{tabular}




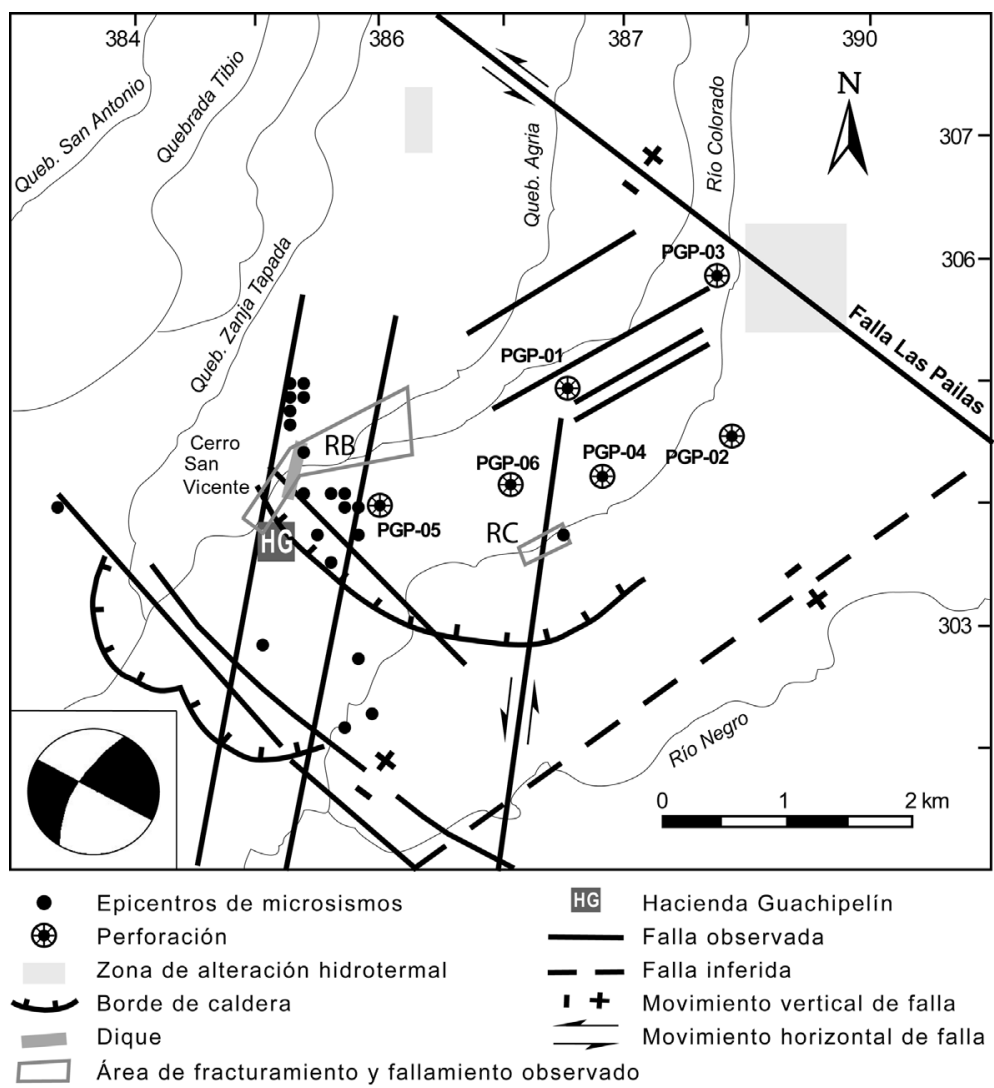

Fig. 2: Localización de los microsismos de mayor amplitud y calidad dentro del área del CGP. Se muestra el mecanismo focal compuesto obtenido usando esos sismos. Los polígonos marcan las áreas del río Blanco (RB) y del río Colorado (RC) donde se observaron fallas y fracturas.

\section{Análisis de los datos de campo}

Una vez identificadas las posibles fallas o zonas de falla activas, se procedió a confirmar su presencia mediante trabajo geológico de campo. Es así como se identificaron diques, fracturas, fallas y paleo-cauces.

\section{Fracturas en el río Blanco y la quebrada Agria}

Basados en la localización de los sismos (especialmente en la figura 2), los cauces de la quebrada Agria y el río Blanco eran los primeros a evaluar para verificar la presencia de fallas y fracturas. Se recorrieron ambos cauces desde la coordenada Lambert este 385,00 hasta la coordenada
387,20 y se pudo observar, en un sector muy restringido (polígono RB, Fig. 2), una serie de fracturas, un dique y un paleocauce (Fig. 3), todos con rumbo predominante de $\mathrm{N} 10-20^{\circ} \mathrm{E}$.

Por lo general, las fracturas (asociadas muy posiblemente con una zona de falla) son abiertas, sin rellenos y afectan todas las capas (especialmente las lavas, tobas y sedimentos de la Unidad Guachipelín). Los sedimentos recientes como los depósitos indeferenciados del volcán Rincón de la Vieja (lahares y coluvios) no se ven afectados por el fallamiento, razón por la cual son difíciles de apreciar en las fotografías aéreas.

Una característica de las fracturas es que aguas abajo de Río Blanco (hacia el este de la intersección del río Blanco y la quebrada Agria), son cada vez más cerradas. El dique observado 

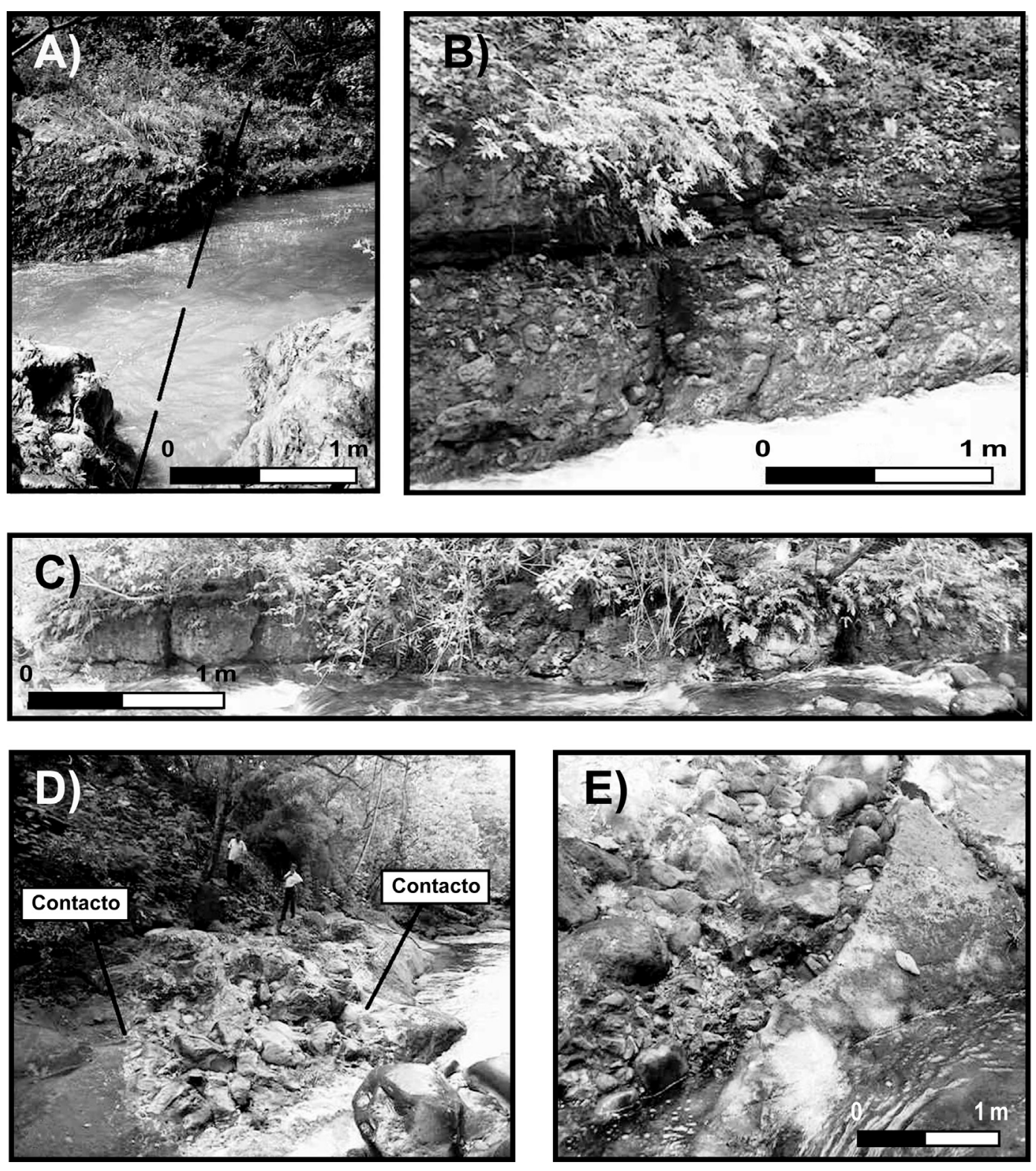

Fig. 3: Fotografías dentro del área RB (Fig. 2). A) fracturas con rumbo N13² E (coordenadas 304,30N/385,80E Lambert Costa Rica Norte), B) fracturas de rumbo $\mathrm{N} 15^{\circ} \mathrm{E}$ (coordenadas 304,30N/385,90E Lambert Costa Rica Norte) que cortan el conglomerado silicificado y la toba en el río Blanco, $\mathrm{C}$ ) fracturas en la margen izquierda de la quebrada Agria con rumbo $\mathrm{N} 15^{\circ} \mathrm{E}$ (coordenadas 304,65N/ 386,00E Lambert Costa Rica Norte), D) dique de $3 \mathrm{~m}$ de ancho con rumbo N20 ${ }^{\circ} \mathrm{E}$ en contacto con tobas en el río Blanco, (coordenadas 304,30N/385,67E Lambert Costa Rica Norte) y E) detalle del contacto entre una toba y un paleocauce con rumbo $\mathrm{N}^{\circ} 0^{\circ} \mathrm{E}$ en la margen derecha del río Blanco (coordenadas 304,30N/385,67E Lambert Costa Rica Norte). 
en el río Blanco posee un rumbo similar al de las fracturas. Esto sugiere que la actividad volcánica (dique y domo San Vicente) se instalaron a lo largo de zonas de debilidad cortical, pudiendo quizás a su vez disminuir la permeabilidad del medio.

\section{Fracturas en el río Colorado}

El patrón de drenaje y las fotografías aéreas son evidencia clara del fuerte control estructural en la zona. Arias (2002) sugiere la presencia de una falla trascurrente sinestral de rumbo NNE justo en el medio del campo geotérmico. Las observaciones de campo (datos estructurales y visuales) así lo confirman. La figura 4 muestra la falla, en cuyas superficies es posible observar "escalones", que sugieren que se trata de una falla de desplazamiento de rumbo sinestral.

Aguas arriba del punto donde fue tomada la foto mostrada en la figura 4, los depósitos recientes (lahares, coluvios, etc.) hacen difícil

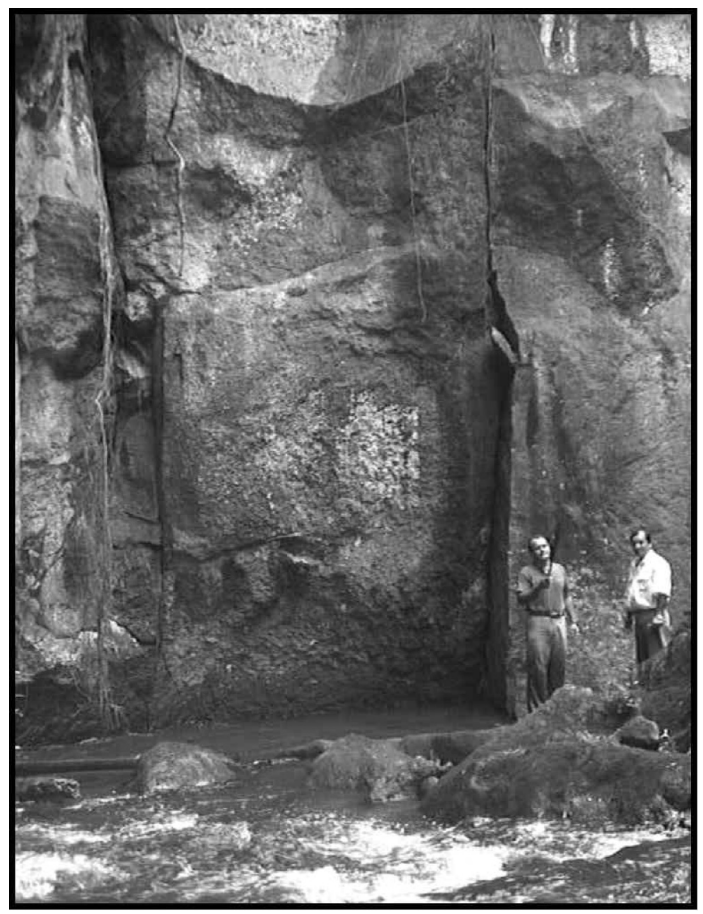

Fig. 4: Falla de rumbo $\mathrm{N} 10^{\circ} \mathrm{E}$ en la margen izquierda del río Colorado (coordenadas 303,75N/387,40E Lambert Costa Rica. la identificación visual de fallas y fracturas, mientras que aguas abajo, el río Colorado se encañona, lo que hace difícil su recorrido. Sin embargo, las evidencias geológicas, sismológicas y estructurales sugieren que de este punto hacia el oeste, las diaclasas, fracturas y fallas predominantes son de rumbo $\mathrm{N} 10^{\circ} \mathrm{E}$.

No se deben olvidar las zonas de debilidad de rumbo $\mathrm{N} 66^{\circ} \mathrm{E}$ que fueron sugeridas por los datos de polarización de las ondas $\mathrm{S}$ y del patrón de drenaje. La existencia de fracturas con ese rumbo preferencial podría verificarse en el cañón del río Colorado, pero para acceder a dicho cañón, se necesita equipo especializado.

\section{Cálculo de las relaciones de Poisson}

Aunque la base de datos sismológica consta de 116 sismos, se eliminaron los valores de $(\sigma)$ que estuvieran fuera del rango 0,1-0,3 debido a que dentro de ese rango se encuentran los valores de $\sigma$ de la mayoría de las rocas de la corteza. De esta forma el número de datos utilizados en este estudio fue de 86 valores.

Los valores de $\sigma$ obtenidos se mantienen principalmente entre 0,20 y 0,30 (69 valores), lo que sugiere que el CGP es un reservorio de alta presión dominado por agua (Fig. 5).

Una vez calculado el valor de $\sigma$ para cada uno de los sismos analizados usando las velocidades de las ondas obtenidos aplicando Wadati (1933), se localizaron estos en el mapa del área para observar su distribución espacial y la distribución de las cuervas de isovalores. Se analizaron los datos para tres diferentes situaciones: todos los datos juntos, los sismos localizados entre 1 y $2 \mathrm{~km}$ de profundidad y los sismos con profundidades mayores a $2 \mathrm{~km}$.

\section{Distribución de $\sigma$ usando todos los datos sin tomar en cuenta la profundidad}

La figura 6 muestra las curvas de isovalores de $\sigma$ obtenidos utilizando todos los sismos sin tomar en cuenta su profundidad, es decir, se utilizaron todos los datos (entre 1,0 y $5,5 \mathrm{~km}$ de 


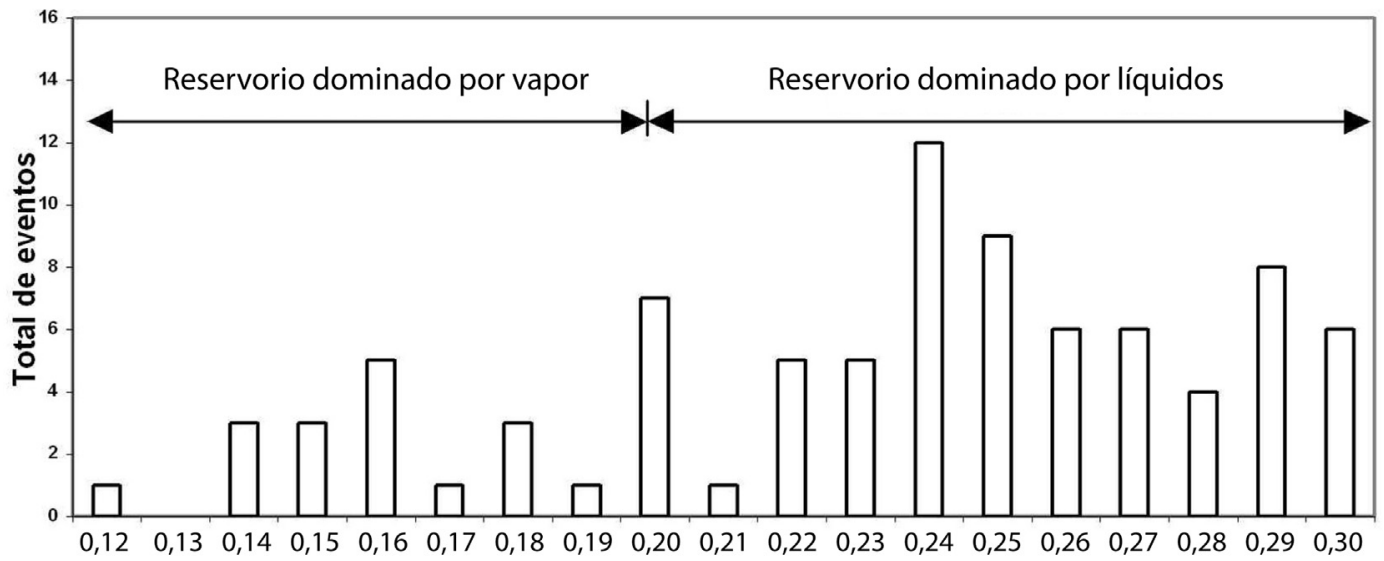

Relación de Poisson $(\sigma)$

Fig. 5: Distribución de los valores de relación de Poisson ( $\sigma$ ) en el CGP usando Wadati (1933), indicando que el campo geotérmico es un reservorio de alta presión dominado por agua.

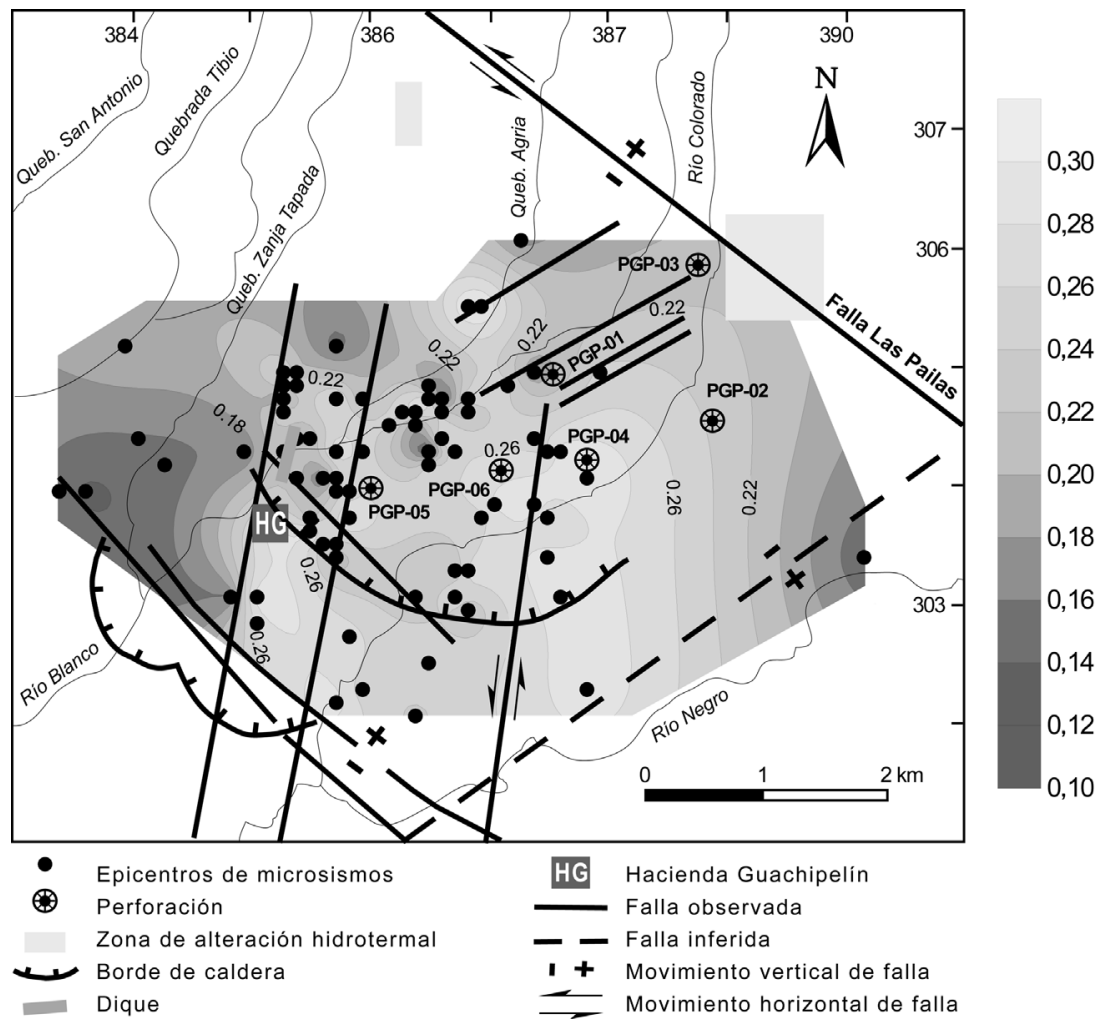

Fig. 6: Mapa de curvas de isovalores de la relación de Poisson ( $\sigma)$. Los tonos más oscuros representan las áreas presumiblemente más calientes (más vapor), los grises intermedios corresponden con las zonas de interacción de fases y los más claros con las zonas relativamente más frías (mayor contenido de líquidos). 
profundidad). En ella se puede apreciar que este campo geotérmico está fuertemente controlado por patrones estructurales y la tectónica. La zona en donde se puede dar la separación de fases (valores cercanos a 0,20 ) se encuentra, por lo general, a lo largo de las zonas de falla, donde se localizan los pozos PGP-01, PGP-03 y PGP-05. Los pozos PGP-04 y PGP-06 se localizan en áreas donde el contenido de líquidos es mayor.

El pozo PGP-02 se encuentra en una zona de separación de fases, pero donde no se ha definido ningún tipo de fallamiento en el sector. Aunque las fotografías aéreas sugieren la existencia de fallas de rumbo NO, y la distribución de las curvas de isovalores de $\sigma$ insinúan algún tipo de estructura de rumbo NS cerca del pozo PGP02, la poca cantidad de datos (un sismo) no permite definir nada en concreto. El oeste del campo está dominado por la presencia de los domos dacíticos en superficie (cerro San Vicente y Sitio Mesas) y posee valores bajos de $\sigma$, por lo que no se descarta la presencia de nuevos cuerpos calientes en profundidad que permitan un mayor contenido de vapor.

\section{Distribución de $\sigma$ en el área del CGP usando los sismos localizados en el rango de $1-2 \mathrm{~km}$ y los mayores a $\mathbf{2} \mathbf{~ k m}$ de profundidad}

Para la confección de estos mapas se utilizaron 63 sismos con profundidades entre los 1 y 2 km (Fig. 7a) y 23 sismos con profundidades entre 2,0 km y 5,5 km (Fig. 7b).

Es evidente en la figura 7 a que no hay mayor cambio con respecto a la figura 6 , debido a que la mayor cantidad de datos corresponden con sismos con profundidades menores o iguales a 2 $\mathrm{km}$. Estas dos figuras (figura 6 y 7a) sugieren que la parte central del campo geotérmico donde se localizan los pozos PGP-04 y PGP-06 es una zona confinada por las fallas existentes y posee un alto contenido de fluidos. Por su parte, la figura $6 \mathrm{~b}$ muestra un área con valores bajos de $\sigma(0,12-0,14)$ que sugieren la existencia de un cuerpo caliente en ese sector por debajo de los $2 \mathrm{~km}$ de profundidad.

\section{DISCUSIÓN}

La distribución espacial de los sismos del enjambre de setiembre del año 2002, los análisis de la orientación de las fracturas (polarización de las ondas S), el mecanismo focal compuesto obtenido, los datos estructurales y las observaciones de campo, permiten definir al menos dos sistemas de fallas activas principalmente de desplazamiento de rumbo, una con rumbo $\mathrm{N}^{\circ} 0^{\circ} \mathrm{O}$ localizada en las cercanías de la Hacienda Guachipelín y la otra con rumbo $\mathrm{N}^{\circ} 6^{\circ} \mathrm{E}$ entre los pozos PGP-05 y PGP-03. Existe un tercer patrón estructural de rumbo $\mathrm{N} 10^{\circ} \mathrm{E}$ que es evidente en los cauces de los ríos Blanco y Colorado y la quebrada Agria, producto del fallamiento principal. Todas estas observaciones sugieren que el CGP está fuertemente influenciado por las estructuras geológicas y por la neotectónica local (sismicidad actual), y es de esperar que durante la explotación del campo se produzcan enjambres similares al ocurrido en setiembre del 2002.

Generalmente, las fallas actúan como barreras impermeables que no permiten el paso de líquidos de un lado a otro, pero sí a lo largo de ellas. El CGP es un reservorio de alta presión dominado por fluidos. El análisis integrado de todos los datos indica que en general se puede dividir el CGP de acuerdo a tres condiciones principales (Fig. 8), cuyas características se detallan a continuación.

\section{Condición con mayor contenido de vapor (Zona I)}

Se localiza en los sectores este y oeste del campo (identificadas con el I, Fig. 8). La caracterización se realizó utilizando las observaciones hechas en el sector oeste, debido a que el sector este no está bien definido por la falta de datos sismológicos y la escasez de observaciones de campo. Son regiones que presentan valores bajos de relaciones de Poisson $(0,12-0,17)$, que normalmente están asociados con regiones donde se da la mayor acumulación de vapor. 


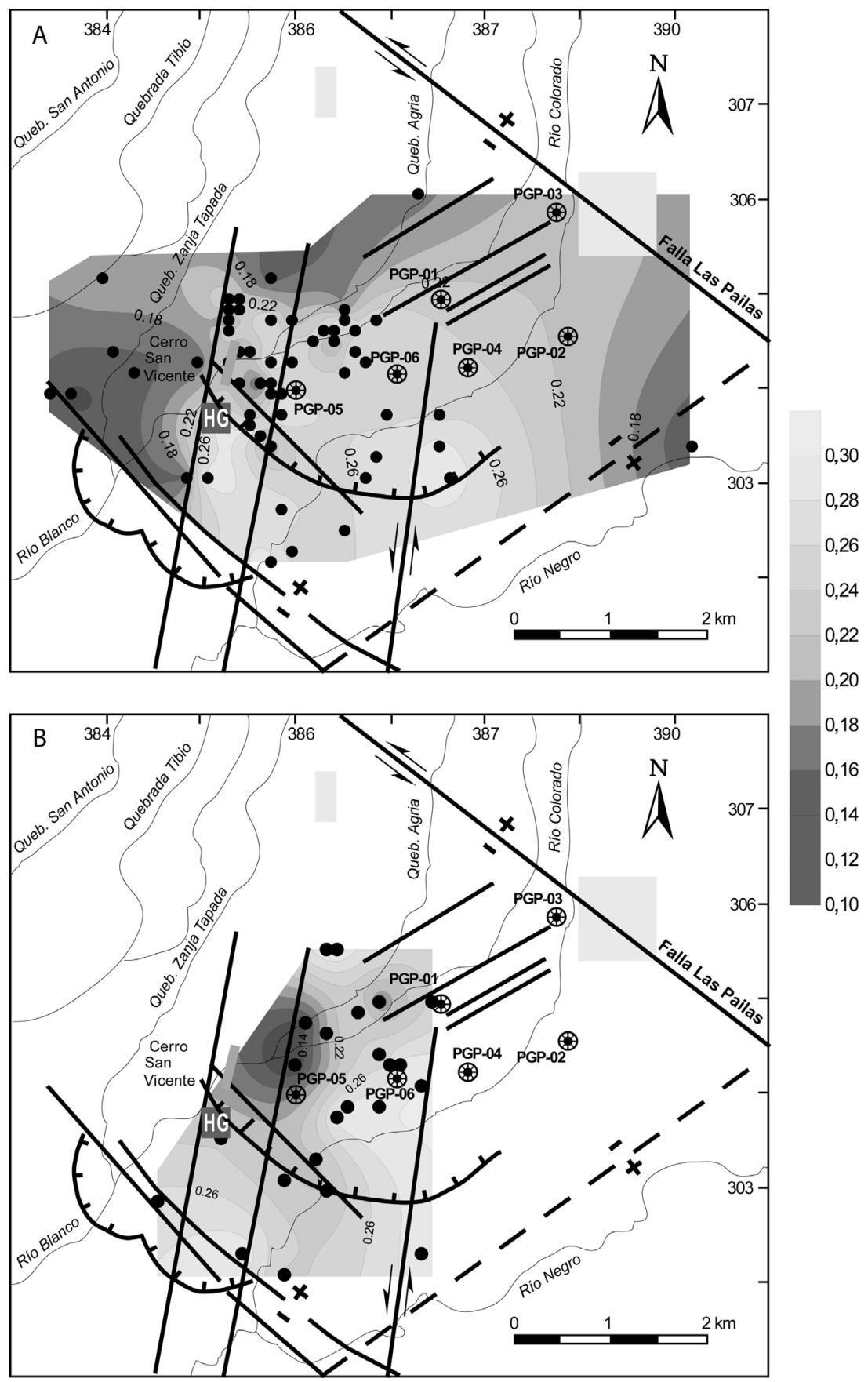

Fig. 7: Mapa de curvas de isovalores de relación de Poisson $(\sigma)$.

A) Sismos con profundidades entre 1 y $2 \mathrm{~km}$.

B) Sismos con profundidades entre 2,0 y $5,5 \mathrm{~km}$. Los colores más oscuros teóricamente representan las áreas más calientes (más vapor) y las más claras las zonas relativamente mas frías (mayor contenido de líquidos). Simbología igual que la figura 1. 


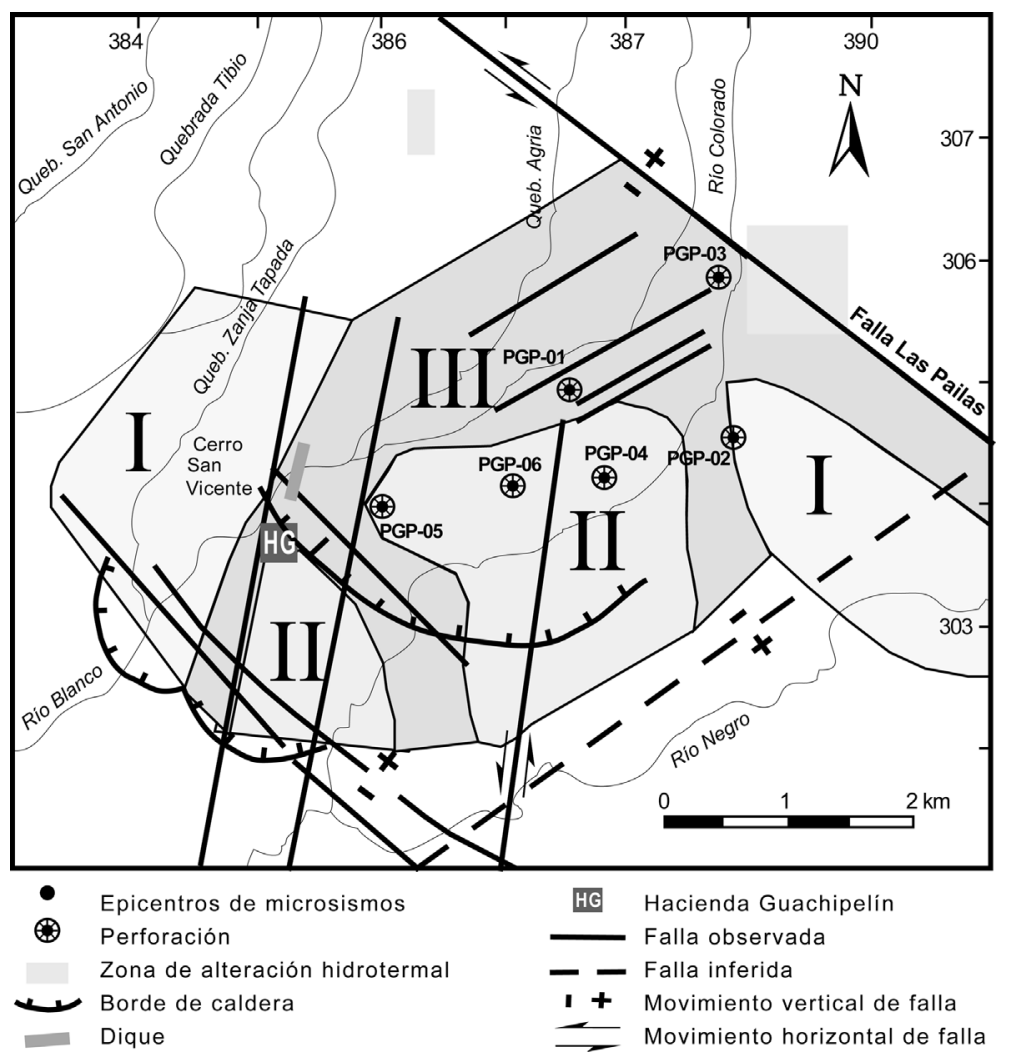

Fig. 8: Distribución espacial de las tres clasificaciones o condiciones definidas preliminarmente en el CGP, utilizando datos sismológicos y observaciones estructurales de campo. La zona I es el área más caliente (mayor contenido de vapor), la zona II la región con mayor contenido de líquidos y la zona III es el área donde se realiza la separación de fases.

Las fallas y fracturas predominantes son de rumbo $\mathrm{N} 11^{\circ} \mathrm{E}$. La existencia del dique y del domo dacítico San Vicente en el sector oeste, sugieren que la permeabilidad podría estar afectada por actividad hidrotermal y puede ser que está sea baja.

\section{Condición con mayor contenido de líquidos (Zona II)}

Se identifica con un II en la figura 8. Tiene valores altos de relaciones de Poisson $(0,24-$ $0,30)$, lo que significa que posee un mayor contenido de líquidos (principalmente agua). Las fracturas y fallas predominantes tienen dos rumbos preferenciales: $\mathrm{N} 11^{\circ} \mathrm{E}$ y $\mathrm{N} 66^{\circ} \mathrm{E}$, pero no se descarta la presencia de fallas de rumbo $\mathrm{NO}$ cerca de los pozos PGP01 y PGP03.

\section{Condición con intercambio de fases (Zona III)}

Son las áreas que se muestran con III en la figura 8 (zona gris). Tienen valores intermedios de relaciones de Poisson $(0,18-0,23)$ y son las zonas de contacto entre las áreas con mayor contenido de vapor y las áreas con mayor contenido de líquidos, es decir, se puede definir como el área donde se produce el cambio de fases. En ella se pueden ubicar los mejores pozos productores 
dependiendo del grado de permeabilidad. Al igual que las otras dos áreas, presenta fracturas $\mathrm{N} 12^{\circ} \mathrm{E}$ y $\mathrm{N} 66^{\circ} \mathrm{E}$ y es muy posible que exista un tercer juego de fracturas con rumbo $\mathrm{N} 70^{\circ} \mathrm{O}$, como es sugerido por los mecanismo focales y las estaciones estructurales realizadas en trabajos anteriores (Arias, 2002).

\section{AGRADECIMIENTOS}

Especiales gracias al personal del OSIVAM, dado que sin ellos la recolección de datos, el mantenimiento de las estaciones sismológicas y el trabajo de campo no se hubiera llevado a cabo con éxito. A las haciendas Guachipelín, Rincón de la Vieja, Borinquen y Las Imágenes, quienes donaron la electricidad y prestaron la infraestructura y el cuido de las estaciones sismológicas. A Guillermo Alvarado y Gerardo J. Soto por la revisión y sugerencias al presente estudio.

\section{REFERENCIAS}

ARIAS O., 2002: Tectocaldera Cañas Dulces-Guachipelín. 45 págs. ICE, UEN PySA, Proyectos y Servicios Asociados, Centro de Servicios de Recursos Geotérmicos, San José [Informe interno].

ASTER, R. C. \& SHEARER, P. M., 1992: Initial shear wave particle motions and stress constraints at the Anza seismic network. - Geophys. J. Internat. 108: 740-748.

BARAHONA, M., BONILLA, E., CORTÉS, R., COTO, L. C., GUZMÁN, G., HERRERA, P., HidAlGO, P., MARTENS, U., MÉNDEZ, J., PÉREZ, K., REYES, K., SJÖBOHM, L., VARGAS, C. \& ZAMORA N., 2001: Geología-vulcanología del campo geotérmico Borinquen-Las Pailas. - 222 págs. Universidad de Costa Rica-Instituto Costarricense de Electricidad [Inf. Campaña Geológica].

HAVSKOV, J. \& OTTEMÖLLER, L., 2000: SEISAN: The earthquake analysis software for Windows, Sunos and Linux, version 7.0. - 227 págs. Univ. Bergen, Noruega.
ITO, H., DeVIBRISS J. \& NUR A., 1979: Compresional and shear waves in saturated rock during water-steam transition. - J. Geophys. Res. 84: 4731-4735.

JULIAN, B. R., ROSS A., FOULGER G. R. \& EVANS J. R., 1996: Three dimensional seismic image of a geothermal reservoir: The Geysers, California. - Geophys. Res. Lett. 23, 685-688.

KANESHIMA, S., 1990: Origin of crustal anisotropy: Shear wave splitting studies in Japan. - J. Geophys. Res. 95: 11121-11133.

KRIGE, D. G., 1966: Two-dimensional weighted moving average trend surfaces for ore valuation. - En: Proc. Symposium on Mathematical Statistics and Computer Aplication in Ore Valuation. Mar. 7-8:Jour. South African Inst. Mining and Metallurgy, Johannesburg:13-38.

MAJER, E. L. \& McEVILLY, T. V., 1979: Seismological investigations at The Geysers geothermal field. Geophysics, 44: 246-269.

MOLINA, F., 2000: Las Pailas geothermal area Rincón de la Vieja Volcano, Costa Rica. - The United Nation Univ. 13: $267-284$.

O’CONNELL, R. J., \& BUDIANSKY, B., 1977: Viscoelastic properties of fluid-satured cracked solids. - J. Geophys. Res. 82: 5719-5735.

PEACOCK, S., CRAMPIN, S., BOOTH, D. C. \& FLETCHER, J. B., 1988: Shear wave splitting in the Anza seismic gap, southern California: Temporal variations as possible precursors. - J. Geophys. Res. 93: 3339-3356.

SCHERBAUM, F., JOHNSON, J. \& REITBROCK, A., 1999: Programmable Interactive Toolbox for Seismological Analysis, PITSA, Version 5.0, 12-02-1999.- 74 págs. Incorporated Research Institutions for Seismology, Data Management Center, Seattle.

TAYLOR, W., 2000: Shear-wave splitting and improved upper crustal velocity model at the Miravalles Geothermal Field, Guanacaste-Costa Rica. - Inst. Solid Earth Physics, Univ. Bergen, Noruega [Tesis M.Sc.].

WADATI, K., 1933: On the travel time of earthquake waves. - Geophys. Mag. 7: 1001-11. 